\title{
Dexmedetomidine attenuates oxidative/nitrative stress in lung tissues of septic mice partly via activating heme oxygenase-1
}

\author{
JINJIN XU ${ }^{1}$, SHAOQING LEI ${ }^{1}$ and GANG YE ${ }^{2}$ \\ ${ }^{1}$ Department of Anesthesiology, Renmin Hospital of Wuhan University, Wuhan, Hubei 430060; \\ ${ }^{2}$ Department of Anesthesiology, The Central Hospital of Enshi Autonomous Perfecture, Enshi, Hubei 445000, P.R. China
}

Received November 18, 2018; Accepted July 19, 2019

DOI: 10.3892/etm.2019.7911

\begin{abstract}
Excessive reactive oxygen/nitrogen species are considered to be one of the primary events that cause lung injury during sepsis. The present study aimed to determine whether dexmedetomidine (Dex) exhibits antioxidative and antinitrative effects on sepsis-induced lung injury and its effect on heme oxygenase-1 (HO-1) activation. The cecal ligation and puncture (CLP) mouse model was used, where male C57BL/6J mice were randomized into groups: Sham, CLP, Dex and Dex + zinc protoporphyrin (ZnPP). Following CLP or sham operation, intraperitoneal injections of $40 \mu \mathrm{g} / \mathrm{kg}$ Dex or saline were administered in the Dex + ZnPP group, intraperitoneal injections of $\mathrm{ZnPP}(40 \mathrm{mg} / \mathrm{kg}$ ) were administered $1 \mathrm{~h}$ prior to the CLP operation. Subsequently, histopathological examination of the lungs and measurement of HO-1 activity in the lung, as well as oxidative and nitrative stress were determined $24 \mathrm{~h}$ following CLP. Dex significantly decreased the levels of oxidative and nitrative stress, as demonstrated by the decreased levels of malondialdehyde and nitrotyrosine, and the protein expression of inducible nitric oxide synthase, as well as increased superoxide dismutase in lung tissues. Also Dex inhibited the elevation of serum interleukin- 6 and tumor necrosis factor- $\alpha$ and increased lung HO-1 activity. Furthermore, the effects of Dex were partially reverted by the HO-1 inhibitor ZnPP. In conclusion, Dex inhibited oxidative/nitrative stress in sepsis and attenuated sepsis-induced acute lung injury partially by increasing HO-1 activity.
\end{abstract}

\section{Introduction}

The third International Consensus defines sepsis as a life-threatening organ dysfunction caused by a dysregulated host response to infection (1). Sepsis-induced injury and

Correspondence to: Dr Gang Ye, Department of Anesthesiology, The Central Hospital of Enshi Autonomous Perfecture, 158 Wuyang Road, Enshi, Hubei 445000, P.R. China

E-mail: 18971282378@163.com

Key words: oxidative stress, nitrative stress, dexmedetomidine, heme oxygenase-1, sepsis dysfunction of multiple organs remain the major cause of death in septic patients. One of the main organ complications of sepsis is the acute lung injury (ALI), which is characterized by alveolar edema, acute hypoxemic respiratory failure and enhanced inflammatory response in the lungs (2).

Although sepsis is a systemic inflammation response syndrome, overwhelming evidence implicates the underlying role of oxidative stress in the pathogenesis of multiple organ failure in septic patients (3). Oxidative stress influences the molecular mechanisms that control inflammation and directly cause tissue damage $(4,5)$. The lung is the primary organ that is affected initially and most severely in sepsis (6). Under normal physiological conditions, there is a balance between the levels of antioxidants and oxidants in the lungs, and a disruption in this balance is considered to be one of the primary events that causes an inflammatory response in the lungs during septic infection $(6,7)$. Furthermore, oxidative stress further activates cytokines and leukocytes, potentially leading to the overexpression of nitric oxide (NO), an excess of which reacts with $\mathrm{O}_{2}^{-}$to produce $\mathrm{ONOO}^{-}$, which mediates nitrative stress in sepsis (8). A clinical study detected a large qaunitity of nitric oxide derived from inducible NO synthase (iNOS) in septic patients, which indicated that nitrative stress is involved in septic-mediated injury (9).

Dexmedetomidine (Dex), a selective agonist of the $\alpha 2$-adrenergic receptor, is an effective sedative, anxiolytic and analgesic agent for critically ill patients. Dex has been reported to exert beneficial effects on respiration during sedation (10). Recent studies on septic animal models revealed that Dex attenuates lung injury (11). However, these studies primarily focused on the effects of Dex on the inflammatory response. To the best of our knowledge, the effects of Dex on oxidative stress and nitrative stress in lung injury have not been reported. Other studies demonstrated that Dex exerted antioxidant effects $(12,13)$. Thus, it was hypothesized that Dex may exert antioxidant and anti-nitrative effects during septic-induced lung injury.

Heme oxygenase-1 (HO-1) is a stress-inducible enzyme that can catalyze the conversion of heme to ferrous iron, carbon monoxide and biliverdin. HO-1 is among the most critical protective mechanisms activated during cellular stress and it is thought to serve a key role in maintaining anti-oxidant/oxidant balance. A previous study revealed that the stimulation of $\mathrm{HO}-1$ was able to exert protective effects against cellular oxidative 
stress (14). Conversely, HO-1 deficient mice presented with major pro-oxidant and pro-inflammatory pathologies (15), and suffered higher mortality rates compared with wild-type mice (16). Furthermore, lung protection by HO-1 was demonstrated in vitro and in vivo in several models of experimental ALI and sepsis (17,18).

The present study was based on the hypothesis that Dex attenuates lung injury and oxidative and nitrative stress in septic mice by activating HO-1.

\section{Materials and methods}

Cecal ligation and puncture (CLP) to induce sepsis in mice. A total of 56 male wild-type mice $(20-25 \mathrm{~g})$ were obtained from the Experimental Centre of Wuhan University (Wuhan, China). The mice were housed in the conditions of $18-22^{\circ} \mathrm{C}$ room temperature and $50-60 \%$ humidity and given free access to standard laboratory diet and drinking water on a 12-h light/dark cycle.

A CLP model was used for the induction of polymicrobial sepsis in mice. As described in a previous study (19), the mice were anesthetized by administering intraperitoneal ketamine hydrochloride $(120 \mathrm{mg} / \mathrm{kg}$ ) and xylazine hydrochloride $(5 \mathrm{mg} / \mathrm{kg})$. The abdominal area was shaved and disinfected. A laparotomy was performed and the cecum was ligated from the top and punctured twice by piercing the cecum with an 18 -gauge needle. A small amount of feces from the bowel was expelled from the puncture hole and the cecum was returned into the peritoneal cavity gently. Sham-operated mice underwent the same procedure but with no ligation and perforation of the cecum. Pre-warmed saline $(0.5 \mathrm{ml} / 100 \mathrm{~g}$ body weight $)$ was injected subcutaneously following surgery. Postoperative pain control was managed with one subcutaneous injection of bupivacaine $(3 \mathrm{mg} / \mathrm{kg})$. All experimental procedures utilizing animals were approved by the Experimental Animal Centre Review Board of Renmin Hospital of Wuhan University (no. WDRM 2018).

Experimental protocol. Mice were randomly divided into four groups: Sham group, CLP group, Dex group (CLP + Dex) and Dex + zinc protoporphyrin (CLP + Dex + ZnPP). Following CLP or sham surgery, intraperitoneal injections of $40 \mu \mathrm{g} / \mathrm{kg}$ Dex or saline were immediately administered once. Znpp IX $(40 \mathrm{mg} / \mathrm{kg})$ was injected via intraperitoneal administered $1 \mathrm{~h}$ before the CLP operation (20). Znpp IX (Sigma-Aldrich; Merck KGaA) was dissolved in $0.2 \mathrm{M}$ sodium hydroxide and adjusted to a pH of 7.4 (21).

Mortality rate. Mice $(\mathrm{n}=40)$ were randomly divided into four groups (10 mice per group). The animals were CLP- or sham-operated and administered Dex $(40 \mu \mathrm{g} / \mathrm{kg})$ (Jiangsu Hengrui Medicine Co., Ltd.) or ZnPP IX ( $40 \mathrm{mg} / \mathrm{kg}$ ) as stated above. Postoperative pain control was managed with subcutaneous injection of bupivacaine $(3 \mathrm{mg} / \mathrm{kg}$, Shandong Hualu Pharmaceutical Co., Ltd.) immediately post-operation once per day. All animals were monitored after the operation and administrations. The time when an animal died from septic infection was recorded as 1 and the time when no death occurred was recorded as 0. SPSS-15.0 software was used to analyze the mortality rate within 96 h. Following 96-h, all experimental animals were euthanized using $100 \% \mathrm{CO}_{2}$ anesthesia using an air displacement rate of $20 \%$ of the chamber volume/min. Preemptive euthanasia was performed for humane reasons if mice showed any of the following signs: Emaciated, gasping, no response to touch or the anal temperature $<25^{\circ} \mathrm{C}$.

Histopathological assessment of pulmonary tissue. Following $24 \mathrm{~h}$ post-CLP surgery, animals were anesthetized by administering intraperitoneal ketamine hydrochloride $(120 \mathrm{mg} / \mathrm{kg})$ and xylazine hydrochloride $(5 \mathrm{mg} / \mathrm{kg})$. The lung tissues were perfused under controlled pressure with PBS at room temperature. The right lung was fixed in $4 \%$ paraformaldehyde at room temperature for $30 \mathrm{~min}$ and then embedded in paraffin, cut into $4 \mu \mathrm{m}$ sections and stained with hematoxylin and eosin respectively for $5 \mathrm{~min}$ at room temperature. The slides were scored under a light microscope (magnification, x200) by two blinded pathologists with expertise in lung pathology. The criteria for scoring lung injury was as follows (22): $0-5$, normal to minimal inflammation; 6-10, mild inflammatory change; 11-15, moderate inflammatory; 16-20, severe inflammatory injury.

Measurement of tissue myeloperoxidase (MPO) activity. MPO is a marker of neutrophil accumulation and activation. MPO activity in lung tissue was measured by using a MPO detection assay kit according to the manufacturer's protocol (Nanjing Jiancheng Bioengineering Institute). Following homogenization and sonication $(25 \mathrm{kHz} ; 4 \times 30 \mathrm{sec})$ of lung tissue at $0^{\circ} \mathrm{C}$, $100 \mu \mathrm{l}$ of supernatant was added to $2.9 \mathrm{ml}$ of o-Dianisidine dihydrochloride and hydrogen peroxide solution for $5 \mathrm{~min}$ at room temperature and then stopped by adding $0.1 \mathrm{ml}$ of hydrochloric acid. Absorbance was measured spectophotometrically at $400 \mathrm{~nm}$ and MPO activity was expressed as U/mg tissue.

Measurement of superoxide dismutase (SOD) activity, malondialdehyde (MDA) levels and total NO production in lung tissue. Following $24 \mathrm{~h}$ post-operation, SOD activity in the lung was measured using a SOD activity assay kit (Nanjing Jiancheng Bioengineering Institute Co., Ltd.; cat. no. A001-3-2) as previously described (14). SOD activity was expressed as $\mathrm{U} / \mathrm{mg}$ protein.

MDA serves as an index of membrane lipid peroxidation. MDA levels in the tissue was determined using a MDA assay kit (Nanjing Jiancheng Bioengineering Institute Co., Ltd.; cat. no. A003-1-2) as previously described (14). The level of MDA was expressed as $\mathrm{U} / \mathrm{mg}$ protein. The content of lung NO was detected with a commercially available NO assay kit (Nanjing Jiancheng Bioengineering Institute Co., Ltd.; cat. no. A012-1-2) according to manufacturer's protocol.

Quantitation of tissue nitrotyrosine content. Lung tissues were homogenized in cold saline. The nitrotyrosine content of lung tissues, a footprint of in vivo $\mathrm{ONOO}^{-}$formation and an index of nitrative stress, was evaluated using a nitrotyrosine ELISA kit (cat. no. ab113848; Abcam) according to the manufacturer's protocol.

Measurement of serum cytokines. Following 24 h post-CLP surgery, $200 \mu \mathrm{l}$ blood was harvested from the heart for the serum interleukin (IL)- 6 and tumor necrosis factor (TNF)- $\alpha$ 
Table I. General characteristics of septic mice.

\begin{tabular}{llc}
\hline A, Sham group & & \\
\hline Characteristics & Baseline & $24 \mathrm{~h}$ \\
\hline Weight $(\mathrm{g})$ & $23.2 \pm 2.1$ & $20.3 \pm 1.8$ \\
Mean blood pressure $(\mathrm{mmHg})$ & $82.0 \pm 1.0$ & $83.0 \pm 6.0$ \\
Temperature $\left({ }^{\circ} \mathrm{C}\right)$ & $37.0 \pm 0.5$ & $37.1 \pm 0.2$ \\
\hline
\end{tabular}

\section{B, CLP group}

\begin{tabular}{lcc}
\hline Characteristics & Baseline & $24 \mathrm{~h}$ \\
\hline Weight $(\mathrm{g})$ & $23.6 \pm 0.7$ & $21.1 \pm 0.8$ \\
Mean blood pressure $(\mathrm{mmHg})$ & $84.0 \pm 2.0$ & $35.0 \pm 6.0^{\mathrm{a}}$ \\
Temperature $\left({ }^{\circ} \mathrm{C}\right)$ & $37.1 \pm 0.1$ & $30.1 \pm 0.2^{\mathrm{a}}$ \\
\hline
\end{tabular}

${ }^{\mathrm{a}} \mathrm{P}<0.05$ vs. baseline. CLP, cecum ligation and puncture.

assays. Serum IL-6 and TNF- $\alpha$ were detected using commercial ELISA kits (cat. nos. DY 506 and DY410-05; R\&D Systems, Inc.) according to manufacturer's protocol.

HO- 1 activity. HO-1 activity was measured $24 \mathrm{~h}$ post-CLP surgery by quantitatively determining biliverdin reductase using a commercial assay kit (Genmed Scientifics, Inc.) as previously described (14). HO-1 activity was expressed as $\mathrm{ng} / \mathrm{mg}$ protein.

Western blot analysis. Protein extraction and western blot analysis was performed as described previously (14). Protein was extracted by radioimmunoprecipitation assay lysis buffer (cat. no. C500005; Sangon Biotech Co., Ltd.) then quantified by bicinchoninic acid method. A total of $50 \mu \mathrm{g}$ of protein was loaded per lane and separated by $4-12 \%$ SDS-PAGE and then transferred to a PVDF membrane. The membrane was blocked by $5 \%$ non-fat milk for $1 \mathrm{~h}$ at room temperature and subsequently incubated with primary antibodies for iNOS $(1: 1,000$; Abcam, cat. nos. ab3523) and GAPDH (1:1,000; Santa Cruz Biotechnology Inc.; cat. nos. sc-32233) overnight at $4^{\circ} \mathrm{C}$. Immunoreactivity was detected by a secondary horseradish peroxidase-conjugated IgG (1:5,000; Thermo Fisher Scientific, Inc.; cat. no. A32731) for $1 \mathrm{~h}$ at room temperature. Proteins were developed via enhanced ECL chemiluminescence reagent kit (cat. no. 32109; Thermo Fisher Scientific, Inc.) and visualized using Bio-rad Imaging systems (Bio-Rad Laboratories, Inc.) and quantified with Quantity One image software (v4.62, Bio-Rad Laboratories).

Statistical analysis. Data were expressed as the mean \pm standard error. Each experiment was repeated for three times. SPSS-15.0 software (SPSS, Inc.) was used for data analysis. Statistical significance was estimated via one-way analysis of variance followed by the Student-Newman-Keul post hoc test. The mortality rates among groups were compared using the Kaplan Meier method, followed by a post hoc test
(Bonferroni's method). $\mathrm{P}<0.05$ was considered to indicate a statistically significant difference.

\section{Results}

General characteristics of septic mice. As presented in Table I, at $24 \mathrm{~h}$ following surgery, CLP mice exhibited severe hypotension with a $58 \%$ decrease in mean blood pressure and hypothermia, which indicated that the CLP model was successfully established.

Pretreatment with Dex improves survival in experimental sepsis. As presented in Fig. 1, the survival rates of mice at $96 \mathrm{~h}$ following surgery were 100, 34.4, 55 and $31.3 \%$ for the sham, CLP, CLP + Dex and CLP + Dex + ZnPP groups, respectively. Compared with the sham group, the mortality rate in the CLP group significantly increased. Dex pretreatment markedly decreased the elevated mortality rate induced by CLP. However, ZnPP reverted the effects of Dex (Fig. 1).

Dex treatment attenuates lung histological injury in sepsis. Representative lung morphological changes are presented in Fig. 2A. Lung tissue sections in sham group showed normal alveolar architecture. In contrast, septic mice exhibited severe lung damage in the CLP group, evidenced by abundant inflammatory cells infiltration, alveolar wall edema and congestion. However, compared with the CLP group, only mild damage in lung tissue sections was observed in the CLP + Dex group, while ZnPP partly revert the effects of Dex. The inflammation scores of lung injury are presented in Fig. 2B. The lung injury score of the CLP + Dex group was significantly lower than that in the CLP group.

Wet/dry weight ratio and MPO activity. The wet/dry weight ratio and MPO activity in the lung tissues of the sham group was low. However, the wet/dry weight ratio and MPO activity of CLP group was significantly higher than those of the sham group (Fig. 3). Pretreatment with Dex significantly inhibited the increase of wet/dry weight ratio and MPO activity in septic mice. However, ZnPP partially reverted the effects of Dex.

Dex reduces CLP-induced pulmonary oxidative injury. A reduction in SOD activity was also observed in septic mice at $24 \mathrm{~h}$ following CLP surgery compared with sham-operated mice (Fig. 4A). MDA content in murine pulmonary tissue was also significantly increased in septic mice compared with sham-operated mice (Fig. 4B). Additionally, Dex treatment significantly decreased pulmonary MDA content and increased pulmonary SOD activity in septic mice. However, $\mathrm{ZnPP}$ reverted the effects of Dex (Fig. 4).

Dex decreases serum levels of proinflammatory cytokines in septic mice. ELISA assays were performed to evaluate the serum levels of IL- 6 and TNF- $\alpha$. Serum levels of TNF- $\alpha$ and IL-6 in septic mice, induced by CLP surgery, were significantly increased compared with those in the sham group. Dex markedly inhibited the sepsis-induced upregulation of TNF- $\alpha$ and IL-6 production, whilst ZnPP reversed the effects of Dex (Fig. 5). 


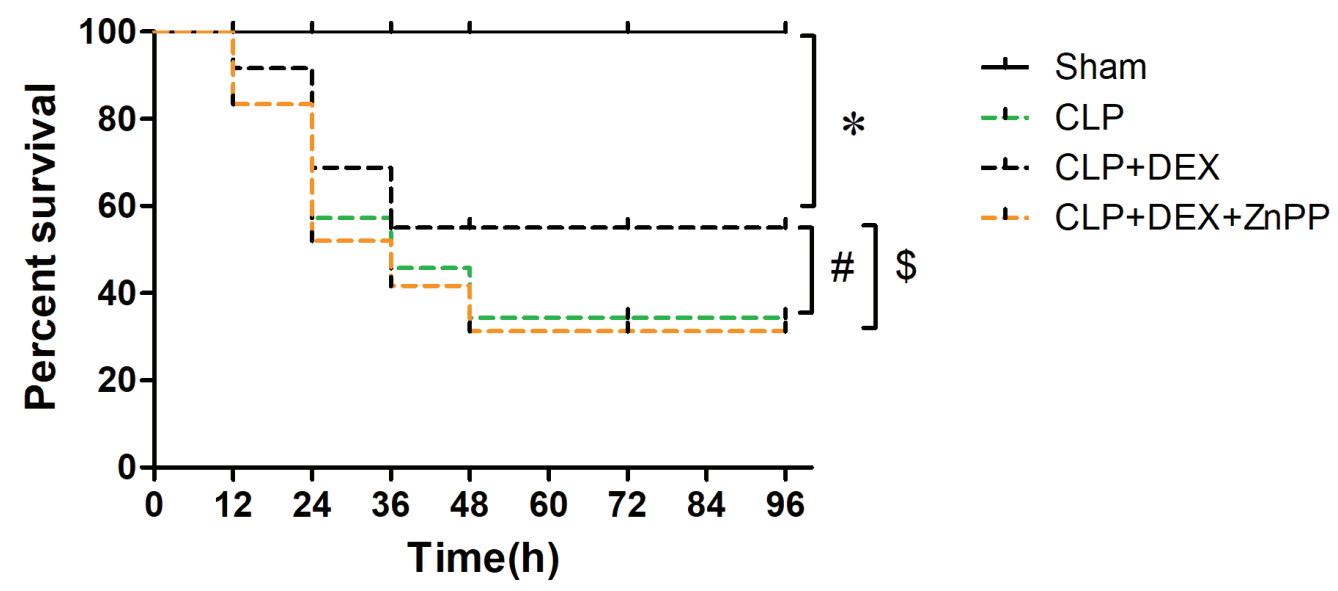

Figure 1. Effect of Dex on the survival rate of septic mice induced by CLP. Sham, sham operation group; CLP, cecal ligation and puncture operation group; CLP + Dex, Dex $(40 \mu \mathrm{g} / \mathrm{kg})$ treatment group; CLP + Dex + ZnPP, CLP with Dex plus ZnPP pre-treatment. ${ }^{*} \mathrm{P}<0.05$ vs. sham, ${ }^{*} \mathrm{P}<0.05$ vs. CLP and ${ }^{\$} \mathrm{P}<0.05$ vs. CLP + Dex. CLP, cecal ligation and puncture; Dex, dexmedetomidine; ZnPP, zinc protoporphyrin.

A i)

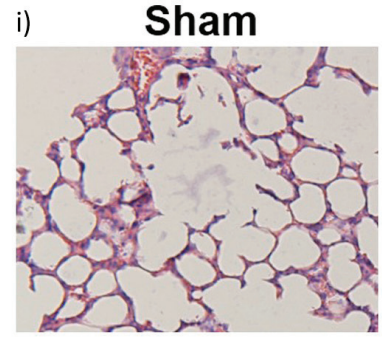

iii) CLP+Dex

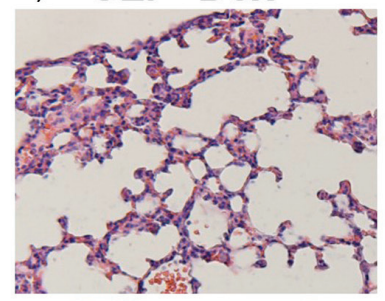

ii) CLP

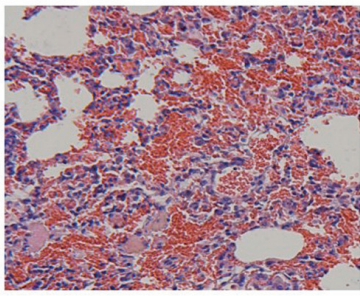

iv) $C L P+D e x+Z n P P$

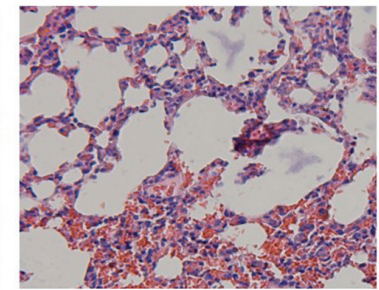

B

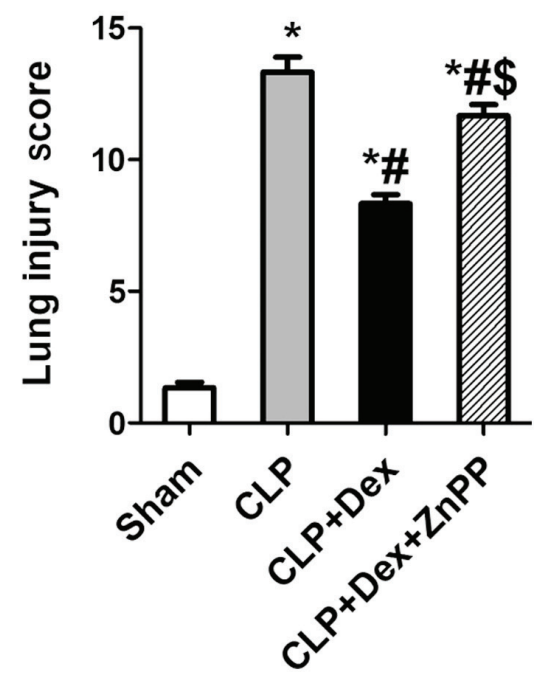

Figure 2. Histological evaluation of lung in septic mice. (A) Representative images of hematoxylin and eosin staining of lung sections from mice in the sham group, CLP group, CLP + Dex group and CLP + Dex + ZnPP group (magnification, $x 200)$. (B) Lung tissue injury scores of mice in the sham, CLP, CLP + Dex and CLP + Dex + ZnPP groups. Data are presented the mean \pm standard deviation ( $\mathrm{n}=6$ per group). ${ }^{\prime \prime} \mathrm{P}<0.05$ vs. sham, ${ }^{*} \mathrm{P}<0.05$ vs. CLP and ${ }^{\$} \mathrm{P}<0.05$ vs. $\mathrm{CLP}+$ Dex. CLP, cecal ligation and puncture; Dex, dexmedetomidine; ZnPP, zinc protoporphyrin.
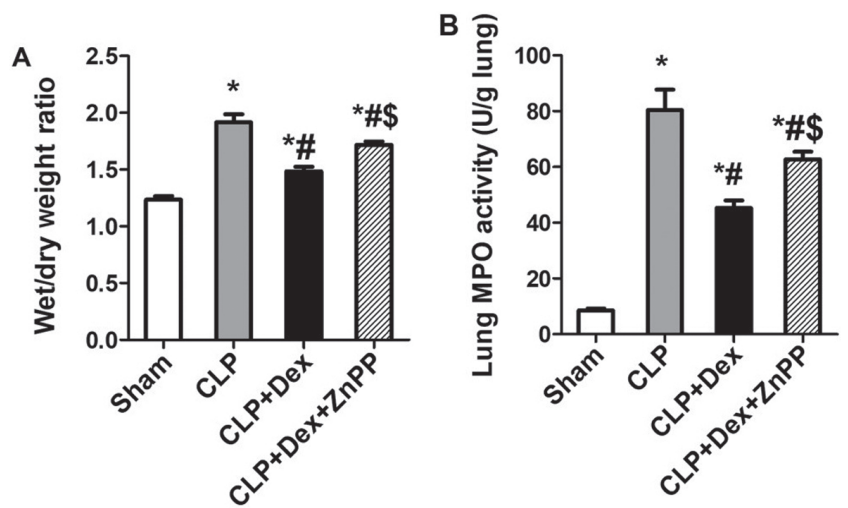

Figure 3. Pulmonary wet/dry weight ratio and MPO activity. (A) Pulmonary wet/dry weight ratio (B) and MPO activity in lung tissues harvested from mice of the sham, CLP, CLP with Dex (CLP + Dex) and CLP with Dex plus $\mathrm{ZnPP}$ pretreatment (CLP + Dex $+\mathrm{ZnPP})$ groups. ${ }^{*} \mathrm{P}<0.05$ vs. sham, ${ }^{*} \mathrm{P}<0.05$ vs. CLP and ${ }^{\$} \mathrm{P}<0.05$ vs. CLP + Dex. Dexmedetomidine; MPO, myeloperoxidase; CLP, cecal ligation and puncture; $\mathrm{ZnPP}$, zinc protoporphyrin.

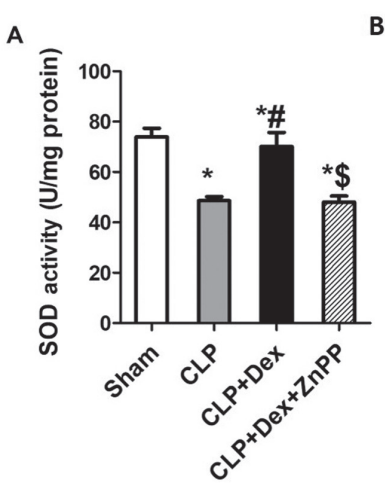

B

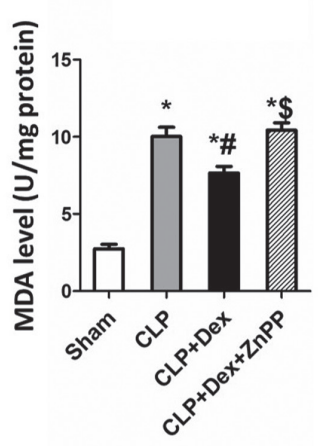

Figure 4. Effect of Dex on lung tissue oxidative stress measured $24 \mathrm{~h}$ after CLP ( $\mathrm{n}=10)$. (A) SOD activity and (B) MDA levels were determined. CLP, cecal ligation and puncture; CLP + Dex, CLP with Dex treatment; CLP + Dex $+\mathrm{ZnPP}, \mathrm{CLP}$ with Dex plus ZnPP pretreatment. ${ }^{*} \mathrm{P}<0.05$ vs. sham, ${ }^{\prime \prime} \mathrm{P}<0.05$ vs. CLP and ${ }^{\mathrm{S}} \mathrm{P}<0.05$ vs. CLP + Dex. Dex, dexmedetomidine; CLP, cecal ligation and puncture; SOD, superoxide dismutase; MDA, malondialdehyde; $\mathrm{ZnPP}$, zinc protoporphyrin. 

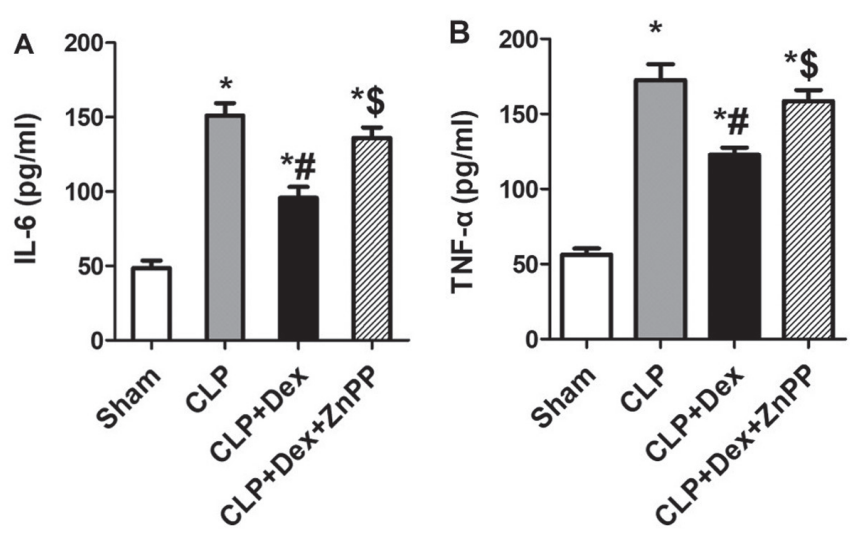

Figure 5. Effect of Dex on the serum levels of (A) IL-6 and (B) TNF- $\alpha$ harvested from mice of the sham, CLP, CLP with Dex treatment and CLP with Dex plus ZnPP pretreatment (CLP + Dex $+\mathrm{ZnPP}) .{ }^{\text {"P }}<0.05$ vs. sham, ${ }^{\sharp} \mathrm{P}<0.05$ vs. CLP and ${ }^{\mathrm{S}} \mathrm{P}<0.05$ vs. CLP + Dex. CLP, cecal ligation and puncture; Dex, dexmedetomidine; TNF, tumor necrosis factor; IL, interleukin; $\mathrm{ZnPP}$, zinc protoporphyrin.

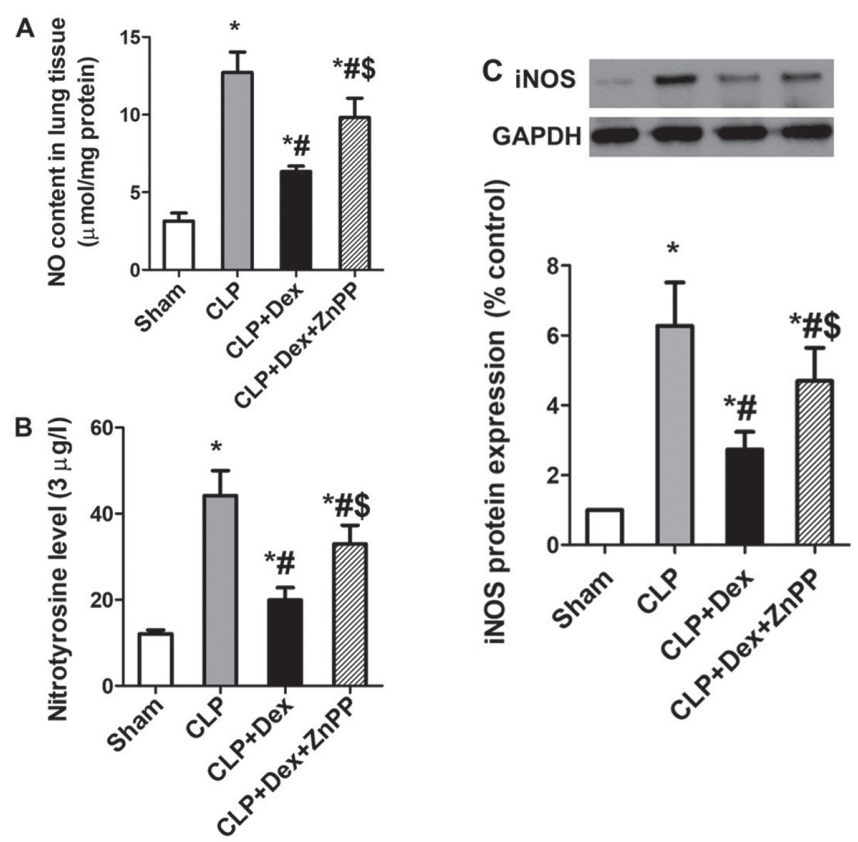

Figure 6. Effect of Dex on nitrative stress in lung tissues harvested from mice of the sham, CLP, CLP with Dex treatment (CLP + Dex), and CLP with Dex plus ZnPP pretreatment (CLP + Dex + ZnPP). (A) Effect of Dex on NO content in lung tissues. (B) Effect of Dex on nitrotyrosine production. (C) Effect of Dex on iNOS protein expression. ${ }^{*} \mathrm{P}<0.05$ vs. sham, ${ }^{,} \mathrm{P}<0.05$ vs. CLP and ${ }^{S} \mathrm{P}<0.05$ vs. CLP + Dex. Dex, dexmedetomidine; NO, nitric oxide; $\mathrm{CLP}$, cecal ligation and puncture; ZnPP, zinc protoporphyrin; iNOS, inducible NO synthase.

Dex decreases CLP-induced pulmonary nitrative injury. Lung tissue nitrotyrosine content was significantly increased in CLP mice compared with the sham-operated group (Fig. 6B). This augmentation was significantly decreased in Dex-treated mice when compared with the CLP group (Fig. 6B). However, ZnPP partially reversed the effects of Dex (Fig. 6B).

NO production was implicated during inflammation, mainly due to the inducible NO synthase (iNOS). NO production and iNOS expression were significantly increased in lung

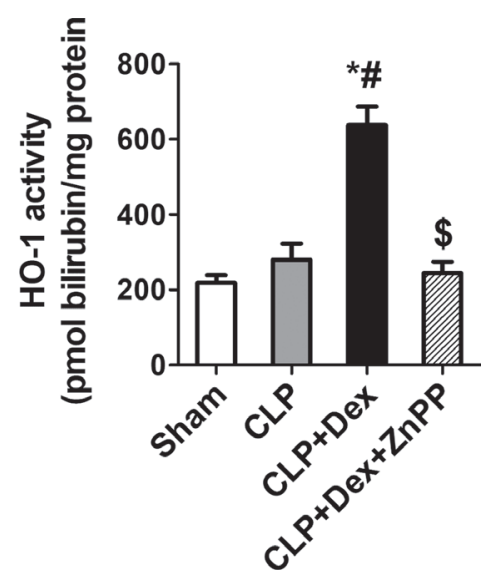

Figure 7. Effect of Dex on HO-1 activity in lung tissues harvested from mice of the sham, CLP, CLP with Dex treatment (CLP + Dex), and CLP with Dex plus $\mathrm{ZnPP}$ pretreatment $(\mathrm{CLP}+\mathrm{Dex}+\mathrm{ZnPP}) .{ }^{*} \mathrm{P}<0.05$ vs. sham, ${ }^{\sharp} \mathrm{P}<0.05$ vs. CLP and ${ }^{\$} \mathrm{P}<0.05$ vs. CLP + Dex. Dex, dexmedetomidine; CLP, cecal ligation and puncture; $\mathrm{ZnPP}$, zinc protoporphyrin; HO-1, heme oxygenase-1.

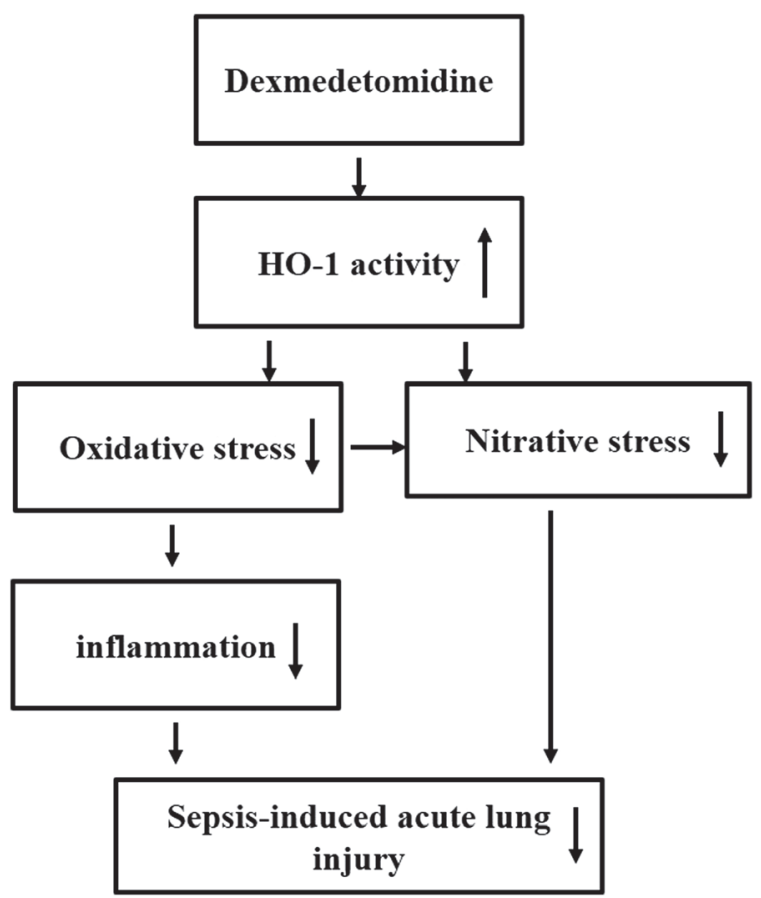

Figure 8. Hypothetic mechanism through which dexmedetomidine attenuates sepsis-induced acute lung injury. HO-1, heme oxygenase-1.

tissues of septic mice (Fig. 6A and C). Dex-treatment significantly decreased $\mathrm{NO}$ production and attenuated the elevated iNOS expression in CLP mice (Fig. 6A and C).

HO-1 activity in lung. HO-1 activity in the lung was low in the sham-operated group. CLP slightly increased the lung HO-1 activity and Dex significantly enhanced HO-1 activity following CLP operation, while ZnPP reversed the effect of Dex (Fig. 7).

\section{Discussion}

The present study revealed that sepsis-induced lung injury was associated with increased oxidative stress and nitrative 
stress, as indicated by a significant increase in lung tissue levels of MDA and nitrotyrosine content. Enhanced levels of oxidative stress were accompanied by compromised plasma SOD activity. Furthermore, Dex prevented septic-induced oxidative/nitrative stress and increased HO-1 activity with concomitant decreased lung levels of MDA, nitrotyrosine and NO content as well as iNOS expression. However, HO-1 inhibitor ZnPP reversed the effects of Dex. The results support the hypothesis that Dex exerts antioxidant and antinitrative effects on septic-induced lung injury partially by increasing HO-1 activity. A hypothetical diagram of this mechanism is presented in Fig. 8.

In sepsis, decreased endogenous antioxidant capacity may lead to the excess production of reactive oxygen species (ROS) $(4,5)$, which have been assumed to serve an important role in the induction of many pro-inflammatory cytokines and mediators to trigger acute inflammatory responses in lung tissue. The antioxidant, $\mathrm{N}$-acetylcysteine, decreases pro-inflammatory cytokine levels and ameliorates sepsis-associated lung injury by suppressing intracellular ROS production (23). Consistent with other reports $(5,6)$ the present study demonstrated that sepsis induced an oxidant-anti-oxidant imbalance, evidenced by significantly increased lung MDA levels and the decreased activity of the antioxidant enzyme, SOD, in septic mice. An increased serum IL- 6 and TNF- $\alpha$ expression and an enhanced lung MPO activity were also observed, which indicated that a large number of inflammatory cells were infiltrating lung tissues. Dex treatment decreased MDA levels, inhibited the expression of IL- 6 and TNF- $\alpha$, and decreased inflammatory cell infiltration, which implied that Dex exerted protective effects on sepsis-induced lung injury by inhibiting oxidative stress. However, the HO-1 ZnPP inhibitor partially reverted the anti-oxidant effects of Dex. The protective effects of Dex against sepsis-induced lung injury was associated with its anti-oxidant property, by directly inhibiting superoxides, but also by indirectly enhancing the activity of anti-oxidant enzyme HO-1.

Sepsis induces lung vascular injury by increasing nitrative stress, which is mediated by an increased NO production, and the formation of strong oxidizing ROS, such as nitrotyrosine $(8,9)$. In the normal lung, NO is mainly produced by endothelial NO synthase, while during inflammation, NO is mainly induced by iNOS (24). Zhang et al (25) reported that resveratrol, which has anti-nitrative property, decreased endotoxemia-induced acute lung injury by decreasing iNOS expression, $\mathrm{NO}$ and nitrotyrosine production. In the present study, the data revealed that septic lungs exhibited a marked increase in nitrative stress, evidenced by the significantly increased NO content and nitrotyrosine production, paralleled with an increased protein expression of iNOS. Dex caused a decreased expression of nitrotyrosine, indicating that Dex attenuates sepsis-induced nitrative stress in lung tissue. However, these effects of Dex were partially reverted by the HO-1 inhibitor ZnPP, suggesting that Dex may exert its anti-nitrative effects by activating the $\mathrm{HO}-1$ pathway.

Enhancing HO-1 activity, leading to the inhibition of oxidative stress/nitrative stress, may be the underlying mechanism whereby Dex attenuates sepsis-induced acute lung injury. HO-1 is a rate-limiting enzyme involved in heme catabolism possessing potent anti-oxidant effects. A number of studies suggested that enhancing $\mathrm{HO}-1$ expression in lung tissue alleviates lung injury induced by sepsis (26-28). Gao et al (29) reported that Dex increased HO-1 expression in lung tissue and decreased oxidative stress during one-lung ventilation. Consistent with this report, the present study determined that Dex significantly increased HO-1 activity, accompanied with decreased MDA levels in the lung tissues of septic mice. However, a previous report has indicated that treatment with an adenoviral vector overexpressing HO-1 inhibits the protein expression of iNOS in the cerebrum and blocks the toxic formation of $\mathrm{ONOO}^{-}$during cerebral ischemia, implying that $\mathrm{HO}-1$ attenuates nitrative stress (8). In the present study, it was demonstrated that Dex markedly activated HO-1 and decreased superoxide anion production and nitrotyrosine content as well as iNOS expression in the lung tissue of septic mice. The HO-1 activity inhibitor partially reverted the effects of Dex, indicating that Dex inhibits sepsis-induced oxidative/nitrative stress to attenuate sepsis-induced lung injury partly by activating HO-1.

In conclusion, Dex was demonstrated to attenuate sepsis-induced acute lung injury by attenuating oxidative/nitrative stress, partially by increasing HO-1 activity. The present results provide further basis for the use of dexmedetomidine to decrease sepsis mortality.

\section{Acknowledgements}

Not applicable.

\section{Funding}

The present study was supported by the National Nature Science Foundation of China (grant no. 81301621) and the National Nature Science Foundation of Hubei, China (grant no. WJ2015MB193).

\section{Availability of data and materials}

All data generated or analyzed during this study are included in this published article.

\section{Authors' contributions}

GY made substantial contributions to conception and design. SL contributed to acquisition of data, analysis and interpretation of data. JX built the model and analyzed data, and was a major contributor in writing the manuscript. All authors read and approved the final manuscript.

\section{Ethics approval and consent to participate}

All experimental procedures utilizing animals were approved by the Experimental Animal Centre Review Board of Renmin Hospital of Wuhan University.

\section{Patient consent for publication}

Not applicable. 


\section{Competing interests}

The authors declare that they have no competing interests.

\section{References}

1. Singer M, Deutschman CS, Seymour CW, Shankar-Hari M, Annane D, Bauer M, Bellomo R, Bernard GR, Chiche JD, Coopersmith CM, et al: The third international consensus definitions for sepsis and septic shock (sepsis-3). JAMA 315: 801-810, 2016.

2. Choudhury S, Kandasamy K, Maruti BS, Addison MP, Kasa JK, Darzi SA, Singh TU, Parida S, Dash JR, Singh V and Mishra SK Atorvastatin along with imipenem attenuates acute lung injury in sepsis through decrease in inflammatory mediators and bacterial load. Eur J Pharmacol 765: 447-456, 2015.

3. Boueiz A and Hassoun PM: Regulation of endothelial barrier function by reactive oxygen and nitrogen species. Microvasc Res 77: 26-34, 2009.

4. Kvietys PR and Granger DN: Role of reactive oxygen and nitrogen species in the vascular responses to inflammation. Free Radic Biol Med 52: 556-592, 2012.

5. Bedreag OH, Rogobete AF, Sarandan M, Cradigati AC, Papurica M, Dumbuleu MC, Chira AM, Rosu OM and Sandesc D: Oxidative stress in severe pulmonary trauma in critical ill patients. Antioxidant therapy in patients with multiple trauma-a review. Anaesthesiol Intensive Ther 47: 351-359, 2015.

6. Lang JD, McArdle PJ, O'Reilly PJ and Matalon S: Oxidant-antioxidant balance in acute lung injury. Chest 122 (6 Suppl): 314S-320S, 2002.

7. Zheng Y and Zhu D: Molecular hydrogen therapy ameliorates organ damage induced by sepsis. Oxid Med Cell Longev 2016: 5806057,2016

8. Chao XD, Ma YH, Luo P, Cao L, Lau WB, Zhao BC, Han F, Liu W, Ning WD, Su N, et al: Up-regulation of heme oxygenase-1 attenuates brain damage after cerebral ischemia via simultaneous inhibition of superoxide production and preservation of NO bioavailability. Exp Neurol 239: 163-169, 2013.

9. Wang Z, Feng K, Yue M, Lu X, Zheng Q, Zhang H, Zhai Y, Li P, Yu L, Cai M, et al: A non-synonymous SNP in the NOS2 associated with septic shock in patients with sepsis in Chinese populations. Hum Genet 132: 337-346, 2013.

10. Becker SE: A pilot study implementing a protocol using dexmedetomidine as a safe alternative to traditional sedation to decrease ventilator days for patients difficult to extubate. Dimens Crit Care Nurs 35: 291-297, 2016.

11. Liu Z, Wang Y, Wang Y, Ning Q, Zhang Y, Gong C, Zhao W, Jing $G$ and Wang Q: Dexmedetomidine attenuates inflammatory reaction in the lung tissues of septic mice by activating cholinergic anti-inflammatory pathway. Int Immunopharmacol 35: 210-216, 2016

12. Xia R, Xu J, Yin H, Wu H, Xia Z, Zhou D, Xia ZY, Zhang L, Li H and Xiao X: Intravenous infusion of dexmedetomidine combined isoflurane inhalation reduces oxidative stress and potentiates hypoxia pulmonary vasoconstriction during One-lung ventilation in patients. Mediators Inflamm 2015: 238041, 2015

13. Ning Q, Liu Z, Wang X, Zhang R, Zhang J, Yang M, Sun $H$, Han F, Zhao W and Zhang X: Neurodegenerative changes and neuroapoptosis induced by systemic lipopolysaccharide administration are reversed by dexmedetomidine treatment in mice. Neurol Res 39: 357-366, 2017.

14. Xu J, Li H, Irwin MG, Xia ZY, Mao X, Lei S, Wong GT, Hung V, Cheung CW, Fang X, et al: Propofol ameliorates hyperglycemia-induced cardiac hypertrophy and dysfunction via heme oxygenase-1/signal transducer and activator of transcription 3 signaling pathway in rats. Crit Care Med 42: e583-e594, 2014.
15. Immenschuh S, Vijayan V, Janciauskiene S and Gueler F: Heme as a target for therapeutic interventions. Front Pharmacol 8: 146, 2017.

16. Wiesel P, Patel AP, DiFonzo N, Marria PB, Sim CU, Pellacani A, Maemura K, LeBlanc BW, Marino K, Doerschuk CM, et al: Endotoxin-induced mortality is related to increased oxidative stress and end-organ dysfunction, not refractory hypotension, in heme oxygenase-1-deficient mice. Circulation 102: 3015-3022, 2000.

17. Yu J, Shi J, Wang D, Dong S, Zhang Y, Wang M, Gong L, Fu Q and Liu D: Heme Oxygenase-1/Carbon Monoxide-regulated mitochondrial dynamic equilibrium contributes to the attenuation of endotoxin-induced acute lung injury in rats and in lipopolysaccharide-activated macrophages. Anesthesiology 125: 1190-1201, 2016.

18. Lee JW, Chun W, Kwon OK, Park HA, Lim Y, Lee JH, Kim DY, Kim JH, Lee HK, Ryu HW, et al: 3,4,5-Trihydroxycinnamic acid attenuates lipopolysaccharide (LPS)-induced acute lung injury via downregulating inflammatory molecules and upregulating HO-1/AMPK activation. Int Immunopharmacol 64: 123-130, 2018.

19. Xu J, Feng Y, Jeyaram A, Jay SM, Zou L and Chao W: Circulating plasma extracellular vesicles from septic mice induce inflammation via MicroRNA- and TLR7-dependent mechanisms. J Immunol 201: 3392-3400, 2018

20. Chen H, Xie K, Han H, Li Y, Liu L, Yang T and Yu Y: Molecular hydrogen protects mice against polymicrobial sepsis by ameliorating endothelial dysfunction via an $\mathrm{Nrf} 2 / \mathrm{HO}-1$ signaling pathway. Int Immunopharmacol 28: 643-654, 2015.

21. Xiong J, Wang K, Yuan C, Xing R, Ni J, Hu G, Chen F and Wang X: Luteolin protects mice from severe acute pancreatitis by exerting HO-1-mediated anti-inflammatory and antioxidant effects. Int J Mol Med 39: 113-125, 2017.

22. Wu Y, Liu Y, Huang H, Zhu Y, Zhang Y, Lu F, Zhou C, Huang L, $\mathrm{Li} X$ and Zhou C: Dexmedetomidine inhibits inflammatory reaction in lung tissues of septic rats by suppressingTLR4/NF-kB pathway. Mediators Inflamm 2013: 562154, 2013.

23. Campos R, Shimizu MH, Volpini RA, de Bragança AC, Andrade L, Lopes FD, Olivo C, Canale D and Seguro AC: $\mathrm{N}$-acetylcysteine prevents pulmonary edema and acute kidney injury in rats with sepsis submitted to mechanical ventilation. Am J Physiol Lung Cell Mol Physiol 302: L640-L650, 2012.

24. van der Vliet A and Cross CE: Oxidants, nitrosants, and the lung. Am J Med 109: 398-421, 2000.

25. Zhang HX, Duan GL, Wang CN, Zhang YQ, Zhu XY and Liu YJ: Protective effect of resveratrol against endotoxemia-induced lung injury involves the reduction of oxidative/nitrative stress. Pulm Pharmacol Ther 27: 150-155, 2014.

26. Cao TH, Jin SG, Fei DS, Kang K, Jiang L, Lian ZY, Pan SH, Zhao MR and Zhao MY: Artesunate protects against sepsis-induced lung injury via heme oxygenase-1 modulation. Inflammation 39: 651-662, 2016.

27. Yu Y, Yang Y, Yang M, Wang C, Xie K and Yu Y: Hydrogen gas reduces HMGB1 release in lung tissues of septic mice in an Nrf2/HO-1-dependent pathway. Int Immunopharmacol 69: 11-18, 2019.

28. Chen X, Wang Y, Xie X, Chen H, Zhu Q, Ge Z, Wei H, Deng J, Xia Z and Lian Q: Heme oxygenase-1 reduces sepsis-induced endoplasmic reticulum stress and acute lung injury. Mediators Inflamm 2018: 9413876, 2018.

29. Gao S, Wang Y, Zhao J and Su A: Effects of dexmedetomidine pretreatment on heme oxygenase-1 expression and oxidative stress during one-lung ventilation. Int J Clin Exp Pathol 8: 3144-3149, 2015.

This work is licensed under a Creative Commons Attribution-NonCommercial-NoDerivatives 4.0 International (CC BY-NC-ND 4.0) License. 